\title{
Decoding the proteomic changes involved in the biofilm formation of Enterococcus faecalis SK460 to elucidate potential biofilm determinants
}

Karthika Suryaletha', Lekshmi Narendrakumar', Joby John², Megha Periyappilly Radhakrishnan', Sanil George and Sabu Thomas ${ }^{1 *}$ (D)

\begin{abstract}
Background: Enterococcus faecalis is a major clinically relevant nosocomial bacterial pathogen frequently isolated from polymicrobial infections. The biofilm forming ability of $E$. faecalis attributes a key role in its virulence and drug resistance. Biofilm cells are phenotypically and metabolically different from their planktonic counterparts and many aspects involved in E. faecalis biofilm formation are yet to be elucidated. The strain E. faecalis SK460 used in the present study is esp (Enterococcal surface protein) and fsr (two-component signal transduction system) negative non-gelatinase producing strong biofilm former isolated from a chronic diabetic foot ulcer patient. We executed a label-free quantitative proteomic approach to elucidate the differential protein expression pattern at planktonic and biofilm stages of SK460 to come up with potential determinants associated with Enterococcal biofilm formation.

Results: The Gene Ontology and Kyoto Encyclopedia of Genes and Genomes (KEGG) enrichment analyses of proteomic data revealed that biofilm cells expressed higher levels of proteins which are associated with glycolysis, amino acid biosynthesis, biosynthesis of secondary metabolites, microbial metabolism in diverse environments and stress response factors. Besides these basic survival pathways, LuxS-mediated quorum sensing, arginine metabolism, rhamnose biosynthesis, pheromone and adhesion associated proteins were found to be upregulated during the biofilm transit from planktonic stages. The selected subsets were validated by quantitative real-time PCR. In silico functional interaction analysis revealed that the genes involved in upregulated pathways pose a close molecular interaction thereby coordinating the regulatory network to thrive as a biofilm community.

Conclusions: The present study describes the first report of the quantitative proteome analysis of an esp and fsr negative non gelatinase producing $E$. faecalis. Proteome analysis evidenced enhanced expression of glycolytic pathways, stress response factors, LuxS quorum signaling system, rhamnopolysaccharide synthesis and pheromone associated proteins in biofilm phenotype. We also pointed out the relevance of LuxS quorum sensing and pheromone associated proteins in the biofilm development of E. faecalis which lacks the Fsr quorum signaling system. These validated biofilm determinants can act as potential inhibiting targets in Enterococcal infections.
\end{abstract}

Keywords: Enterococcus faecalis, Biofilm determinants, Quantitative proteomics, Metabolic pathways, Stress response, luxS

\footnotetext{
* Correspondence: sabu@rgcb.res.in

${ }^{1}$ Cholera and Biofilm Research Laboratory, Pathogen Biology, Rajiv Gandhi

Centre for Biotechnology, (National Institute under the Department of

Biotechnology, Government of India), Trivandrum, Kerala 695014, India

Full list of author information is available at the end of the article
}

(c) The Author(s). 2019 Open Access This article is distributed under the terms of the Creative Commons Attribution 4.0 International License (http://creativecommons.org/licenses/by/4.0/), which permits unrestricted use, distribution, and reproduction in any medium, provided you give appropriate credit to the original author(s) and the source, provide a link to the Creative Commons license, and indicate if changes were made. The Creative Commons Public Domain Dedication waiver (http://creativecommons.org/publicdomain/zero/1.0/) applies to the data made available in this article, unless otherwise stated. 


\section{Background}

Enterococci have emerged as a global cause of nosocomial infection and are frequently associated with chronic ulcers, urinary tract infections, endocarditis and indwelling medical device-related infections. The robust biofilm formation and inherent multidrug resistance in Enterococci have uplifted it as a challenging nosocomial pathogen. Biofilms are cell populations irreversibly attached to surfaces and encased within a self-produced hydrated matrix of exopolymeric substances, proteins, polysaccharides, extracellular DNA (eDNA) and water channels. Biofilm confers high antibiotic tolerance ability and resistance to phagocytosis and hence play a prime role in critical colonization, establishment and chronicity of infections. This renders Enterococci to be recalcitrant towards the current treatment strategies. Owing to its egressing clinical significance, the pace of enterococcal biofilm research has accelerated in the past few years for providing a better understanding of intricate underlying mechanisms of biofilm formation.

In E.faecalis, fsr two-component signal transduction system is a well-defined quorum sensing system which regulates biofilm formation by gelatinase production [1]. Previous studies on enterococcal biofilm have suggested several other biofilm associated factors including enterococcal surface protein (esp) [2], aggregation substance [3], pili [4], autolysin [5] and D-alanylation of Lipoteichoic acid (dltABCD) [6]. Several studies on E. faecalis comparing biofilm formers and non-biofilm formers have identified protein translation machinery, aromatic amino acid biosynthesis and sugar and sulfate permease transporter systems to have a momentous role in biofilm formation [7, 8]. E. faecalis SK460 used in the present study is isolated from a chronic diabetic ulcer patient [9] and is devoid of several well-defined biofilm associated factors including fsr quorum signaling, gelatinase production and enterococcal surface protein. Lack of these biofilm determinants does not affect the biofilm forming potential of SK460. This led us to focus on the role of differential protein expression pattern in biofilm phenotype of this strong biofilm former. The present study utilized label-free quantitative approach to decipher the protein expression pattern of E. faecalis SK460 at planktonic and biofilm stages to elucidate the unexplored links in understanding the enterococcal biofilms. This helps to deliver the comprehensive knowledge regarding the metabolic pathways and cellular processes involved in Enterococcal biofilm to come up with potential biofilm inhibiting targets.

\section{Results}

Biofilm forming potential of E. faecalis SK460

Confocal Laser Scanning Microscopy analysis evidenced the high biofilm forming ability of E. faecalis SK460 (Fig. 1). The $24 \mathrm{~h}$ old biofilm showed an average thickness of approximately $40 \mu \mathrm{m}$.

\section{Proteome profile obtained in planktonic and biofilm stages}

Label-free quantitative proteomics identified 657 proteins from planktonic stages and 553 proteins from biofilm stages. Of these, 233 (29.6\%) and 129 (16.4\%) proteins were identified exclusively in the planktonic and biofilm stages respectively and 424 (53.9\%) proteins were detected at both stages.

\section{Physico-chemical properties of identified proteome}

The hydrophobic nature of the identified proteins was calculated using the GRAVY (grand average hydropathy) tool and the score obtained ranges between -1.6 and 1 (Additional file 2: Figure S1a). Nearly $88 \%$ of the proteins were hydrophilic $(<0)$ and the rest were hydrophobic or membranous $(>0)$ in nature. The compute $\mathrm{pI} /$ MW tool revealed that the extracted proteins were within the pI range of 3.5-11.5 and molecular weight of $4-186 \mathrm{kDa}$ (Additional file 3: Figure S1b). $90 \%$ of the proteins were within the range of $80 \mathrm{kDa}$.

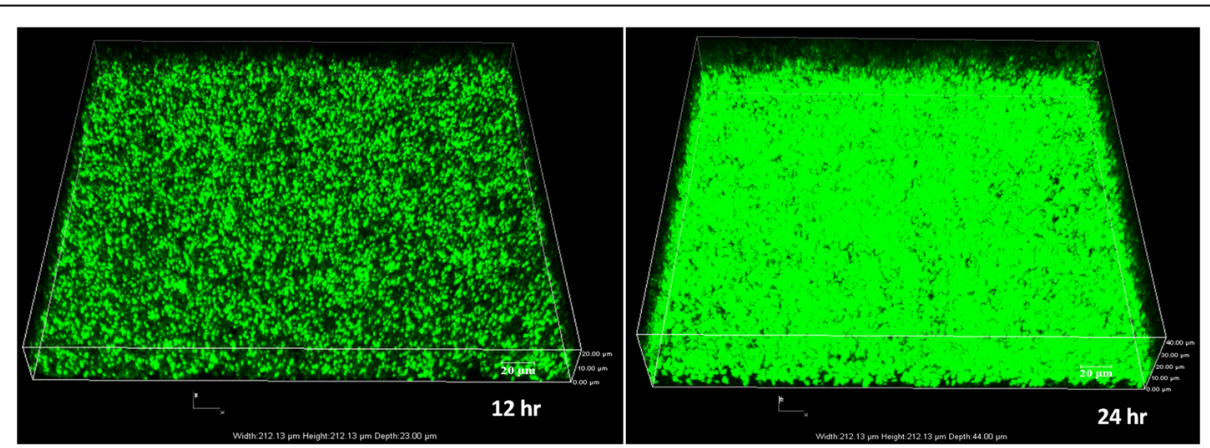

Fig. 1 Confocal laser scanning microscopy images of biofilm formation of E. faecalis SK460 at $12 \mathrm{~h}$ and $24 \mathrm{~h}$. The images are processed using NISElement AR software, version 4.00.04 
Functional categorization of proteome at planktonic and biofilm stages

\section{KEGG pathways}

The proteins identified in the planktonic and biofilm stages were assigned to define KEGG pathways. The major pathways were associated with ribosome (72 hits), pyruvate metabolism (27 hits), pyrimidine metabolism (50 hits), amino acid biosynthesis (69 hits) and antibiotics (122 hits), Fatty acid metabolism (15 hits), RNA degradation (13 hits), biosynthesis of secondary metabolites (157 hits), glycolysis/gluconeogenesis (40 hits), citrate cycle (TCA cycle) (7 hits), microbial metabolism in diverse environments (110 hits), propanoate metabolism (11 hits) and butanoate metabolism ( 9 hits). Furthermore, the proteins upregulated/unique to biofilm stages were assigned to KEGG pathways and revealed the enrichment of glycolysis/gluconeogenesis, pyruvate metabolism, biosynthesis of secondary metabolites, microbial metabolism in diverse environments and arginine metabolism (Fig. 2). More than $50 \%$ of the total proteins involved in these pathways were detected to be upregulated in biofilm stages of SK460.

\section{Biological processes}

The proteins were clustered based on gene ontology (GO) analysis and its relative expression at planktonic and biofilm stages were analyzed. Comparative proteome analysis revealed that several ribosomal proteins and proteins involved in DNA replication and transcription were found to be normally expressed or slightly upregulated. The relative abundance of some proteins involved in nucleotide metabolism and protein biosynthesis was affected to differing degrees under biofilm conditions. Major functional categories of proteins upregulated in biofilm stages are listed in Table 1.

In general, major glycolytic enzymes and several enzymes in the citric acid cycle and pentose phosphate pathway were found to be in higher abundance in biofilm stages of SK460. Enzymes involved in rhamnose biosynthesis which forms the part of enterococcal polysaccharide antigen gene cluster was also found to be upregulated in biofilm stages. Compared to planktonic forms, Enterococcal biofilms showed a hike in stress response factors, DNA repair system, molecular chaperones, pheromone associated lipoproteins and other adhesion associated proteins. Besides, the present proteome analysis established the role of $\operatorname{luxS}$ mediated quorum sensing in enterococcal biofilm development.

STRING analysis predicted the molecular interactions among the highly expressed proteins in biofilm stages with enrichment $p$-value $<1.0 \mathrm{e}-16$ and is shown in Fig. 3. The functional interactome of the whole upregulated protein sets is depicted in Additional file 2: Figure S2.

Validation of selected biofilm associated genes by RT-PCR The genes $r f b B$ (Rhamnose biosynthesis), $\operatorname{arcA}$ (Arginine deiminase), luxS (Quorum sensing), $c A D 1$ (Pheromone

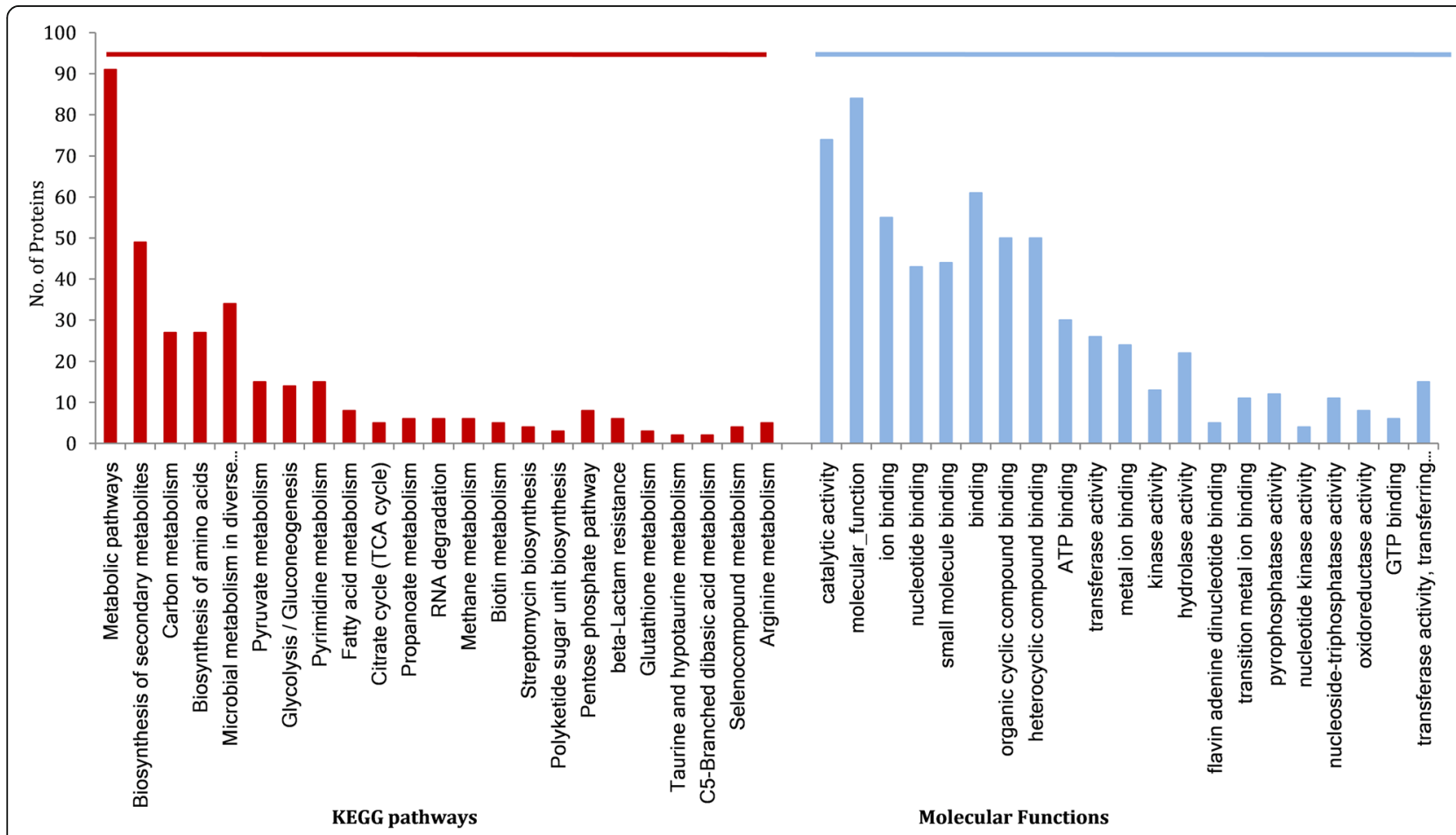

Fig. 2 KEGG pathways and molecular functions assigned to categorize the upregulated proteins obtained in biofilm stages of E. faecalis SK460 
Table 1 List of proteins enhanced in biofilm stages of E. faecalis SK460

\begin{tabular}{|c|c|}
\hline NCBI-ID & Pathways/cellular processes \\
\hline \multicolumn{2}{|c|}{ Amino acid metabolism } \\
\hline NP_816782.1 & Succinyl-diaminopimelate desuccinylase (EF_3178) \\
\hline NP_816328.1 & MTA/SAH nucleosidase (pfs) \\
\hline NP_815300.1 & Cysteine synthase A (cysK) \\
\hline NP_815281.1 & Chorismate synthase (aroC) \\
\hline NP_815821.1 & Glutamine synthetase, type I $(g \ln A)$ \\
\hline NP_814529.1 & S-adenosylmethionine synthetase (metK) \\
\hline NP_815283.1 & 3-phosphoshikimate 1-carboxyvinyltransferase (aroA) \\
\hline NP_816193.1 & Serine hydroxymethyltransferase ( $g l y A)$ \\
\hline NP_816069.1 & Threonine synthase (thrC) \\
\hline NP_815279.1 & 3-deoxy-7-phosphoheptulonate synthase (EF_1562) \\
\hline NP_816700.1 & Bifunctional glutamate--cysteine ligase/glutathione synthetase (gshF) \\
\hline NP_816650.1 & Glutamyl-aminopeptidase (pepA) \\
\hline NP_814940.1 & Acetolactate synthase (EF_1213) \\
\hline NP_815294.1 & Dihydrofolate reductase $(f \circ / A)$ \\
\hline \multicolumn{2}{|c|}{ Glycolysis, citric acid cycle, pentose phosphate pathway } \\
\hline NP_815638.1 & Triosephosphate isomerase (tpiA) \\
\hline NP_815639.1 & Phosphoglycerate kinase (pgk) \\
\hline NP_814897.1 & Fructose-bisphosphate aldolase (fba) \\
\hline NP_813994.1 & Phosphoglycerate mutase (gpmA) \\
\hline NP_815245.1 & Glyceraldehyde 3-phosphate dehydrogenase (gap-1) \\
\hline NP_815637.1 & Enolase (eno) \\
\hline NP_815137.1 & Glucose-6-phosphate isomerase (pgi) \\
\hline NP_815075.1 & Pyruvate dehydrogenase complex, E1 component subunit beta ( $p d h B)$ \\
\hline NP_815076.1 & Dihydrolipoamide acetyltransferase (acef) \\
\hline NP_816104.1 & Pyruvate carboxylase $(p y c A)$ \\
\hline NP_813976.1 & Deoxyribose-phosphate aldolase (deoC) \\
\hline NP_813986.1 & Phosphopentomutase (deoB) \\
\hline NP_814782.1 & 6-phosphogluconate dehydrogenase (gnd) \\
\hline \multicolumn{2}{|c|}{ Stress response, chaperones and DNA repair } \\
\hline NP_814237.1 & OsmC/Ohr family protein (EF_0453) \\
\hline NP_816831.1 & Dps family protein (EF_3233) \\
\hline NP_816649.1 & Thioredoxin family protein (EF_3036) \\
\hline NP_814938.1 & NADH peroxidase $(n p r)$ \\
\hline NP_815447.1 & General stress protein (EF_1744) \\
\hline NP_816369.1 & Alkyl hydroperoxide reductase subunit C (ahpC) \\
\hline NP_813885.1 & gls24 protein \\
\hline NP_816648.1 & Universal stress protein (EF_3035) \\
\hline NP_816867.1 & Glutathione reductase (gor) \\
\hline NP_816700.1 & Bifunctional glutamate--cysteine ligase/glutathione synthetase (gshF) \\
\hline NP_816368.1 & Thioredoxin reductase/glutathione-like protein (EF_2738) \\
\hline NP_814049.1 & L-lactate dehydrogenase (Idh 1 ) \\
\hline NP_816550.1 & AhpC/TSA family protein (EF_2932) \\
\hline NP_815246.2 & GTPase ObgE \\
\hline
\end{tabular}


Table 1 List of proteins enhanced in biofilm stages of E. faecalis SK460 (Continued)

\begin{tabular}{|c|c|}
\hline NCBI-ID & Pathways/cellular processes \\
\hline NP_815059.1 & Thioredoxin reductase $(\operatorname{tr} x B)$ \\
\hline NP_815353.1 & Transcriptional repressor CodY (codY) \\
\hline NP_816010.1 & ATP-dependent Clp protease, ATP-binding protein ClpB ( $c \mid p B)$ \\
\hline NP_814518.1 & ATP-dependent Clp protease proteolytic subunit ( $C / p P$ ) \\
\hline NP_816516.1 & Cyclophilin type peptidyl-prolyl cis-trans isomerase (EF_2898) \\
\hline NP_814457.1 & ATP-dependent Clp protease, ATP-binding protein ClpE ( $c \mid p E$ ) \\
\hline NP_816532.1 & Transcription elongation factor GreA \\
\hline NP_815030.1 & dnak protein \\
\hline NP_815032.1 & dnaJ protein \\
\hline NP_815029.1 & Heat shock protein GrpE (grpE) \\
\hline NP_814465.1 & Trigger factor $(T \mid G)$ \\
\hline NP_816273.1 & GroS (10 kDa chaperonin) \\
\hline NP_816272.1 & GroL(60 kDa chaperonin) \\
\hline NP_814060.1 & Fn1610 (33 kDa chaperonin) (hs/O) \\
\hline NP_814686.1 & Uracil-DNA glycosylase (ung) \\
\hline NP_814720.1 & DNA repair protein $\operatorname{RecN}(r e c N)$ \\
\hline \multicolumn{2}{|c|}{ Rhamnose biosynthesis } \\
\hline NP_815854.1 & dTDP-glucose 4,6-dehydratase $(r f b B)$ \\
\hline NP_815853.1 & dTDP-4-dehydrorhamnose reductase (EF_2191) \\
\hline NP_815856.1 & Glucose-1-phosphate thymidylyltransferase (rfbA) \\
\hline \multicolumn{2}{|c|}{ Arginine metabolism } \\
\hline NP_813908.1 & Ornithine carbamoyltransferase $(\operatorname{arc} B)$ \\
\hline NP_815423.1 & Carbamoyl phosphate synthase small subunit (carA) \\
\hline NP_814373.1 & Ornithine cyclodeaminase (EF_0616) \\
\hline NP_813909.1 & Carbamate kinase $(\operatorname{arcC} 1)$ \\
\hline NP_813907.1 & Arginine deiminase $(\operatorname{arc} A)$ \\
\hline NP_814719.1 & ArgR family transcriptional regulator (EF_0983) \\
\hline \multicolumn{2}{|c|}{ Quorum sensing and pheromone associated lipoprotein } \\
\hline NP_814911.1 & S-ribosylhomocysteinase (luxS) \\
\hline NP_816853.1 & Pheromone CAD1 lipoprotein (EF_3256) \\
\hline NP_814793.1 & Pheromone binding protein (EF_1060) \\
\hline NP_815061.1 & Pheromone cAM373 lipoprotein (EF_1340) \\
\hline \multicolumn{2}{|c|}{ Adhesion associated proteins } \\
\hline NP_814266.1 & Aggregation substance (EF_0485) \\
\hline NP_814342.1 & Adhesion lipoprotein (EF_0577) \\
\hline NP_814975.1 & Fibronectin/fibrinogen binding protein (EF_1249) \\
\hline \multicolumn{2}{|c|}{ Autolysin and D-Alanylation of teichoic acid } \\
\hline NP_814543.1 & Autolysin (EF_0799) \\
\hline NP_816376.1 & D-alanine--poly(phosphoribitol) ligase subunit 2 (dlt C) \\
\hline NP_816375.1 & dltD protein $(d l t D)$ \\
\hline
\end{tabular}

cAD1 lipoprotein) and $f b n$ (Fibrinogen/Fibronectin binding protein associated with adhesion) were selected to monitor the fold change expression in biofilm stage when compared to planktonic stage and the results are shown in Fig. 4. All these five genes were found to be upregulated and adhesion protein $(f b n)$ showed a comparatively large fold increase in expression. This substantiates the significant role of these pathways in the development of mature biofilm. 


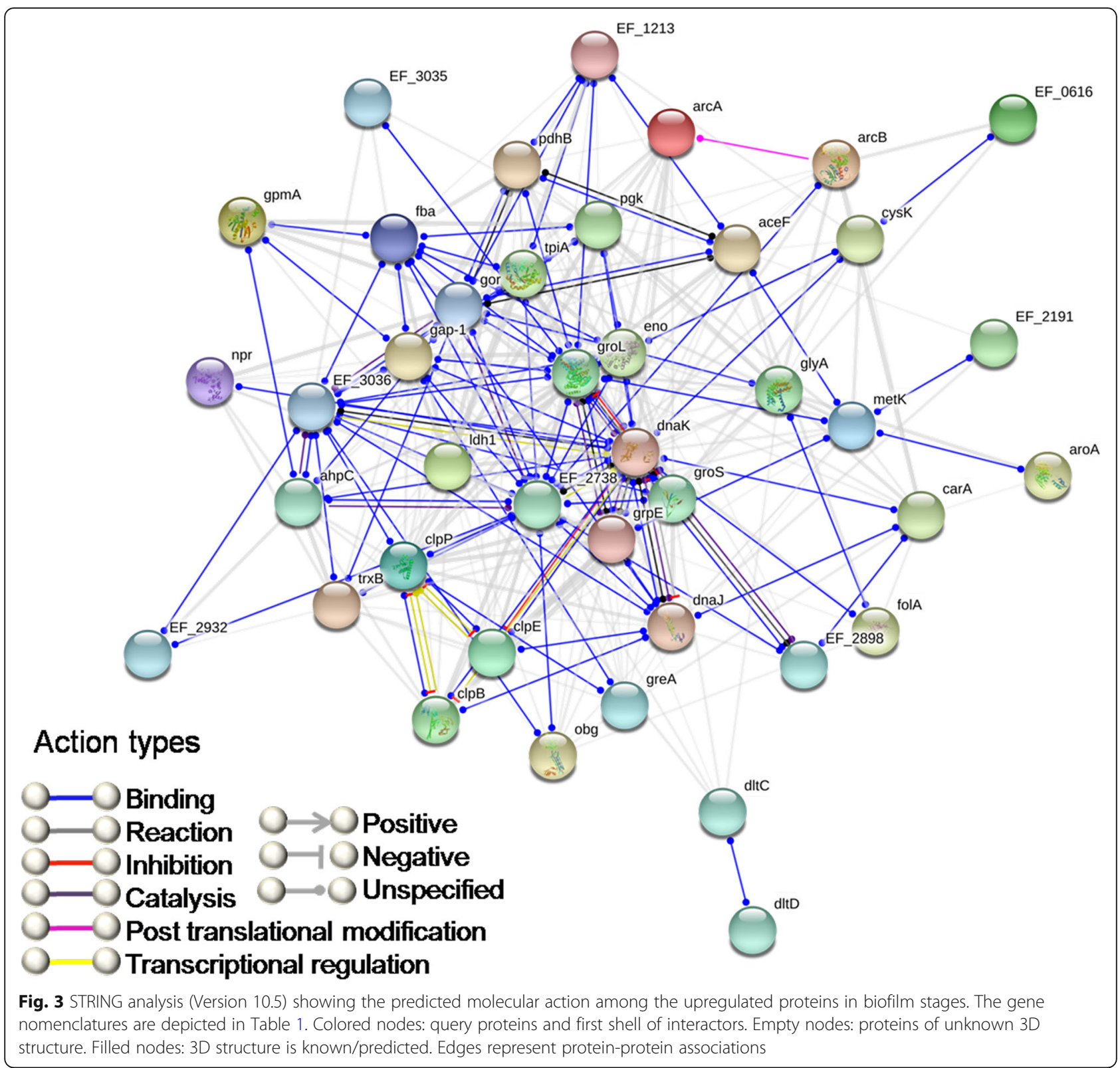

\section{Discussion}

E. faecalis is a clinically relevant hospital associated pathogen and the recalcitrant enterococcal biofilms are known to play a foremost role in establishing chronic infections. The wound isolate E. faecalis SK460 was proved to be a robust biofilm former by confocal laser scanning microscopy. Though SK460 is a potent biofilm former, it is devoid of several well-known biofilm determinants including fsr quorum signaling system and gelatinase production. Previous studies have established the role of fsr regulated gelatinase production and enterococcal surface protein in Enterococcal biofilm development. Besides these aforementioned determinants, several other factors are yet to be elucidated to pick out the unidentified factors associated with enterococcal biofilm. Hence, our prime focus was to decipher the metabolic pathways and physiological processes related to the Enterococcal biofilm development utilizing the label-free quantitative proteomic approach.

In the present investigation, the majority of proteins involved in basic survival mechanisms including DNA replication and transcription were either normally expressed or slightly upregulated in biofilm stages. Starting from the planktonic form to microcolonies and maturing biofilm, an intracellular pool of carbohydrates, amino acids and lipids is required to facilitate the development of biomass required for the sessile growth period. Besides these, certain putative transcriptional 


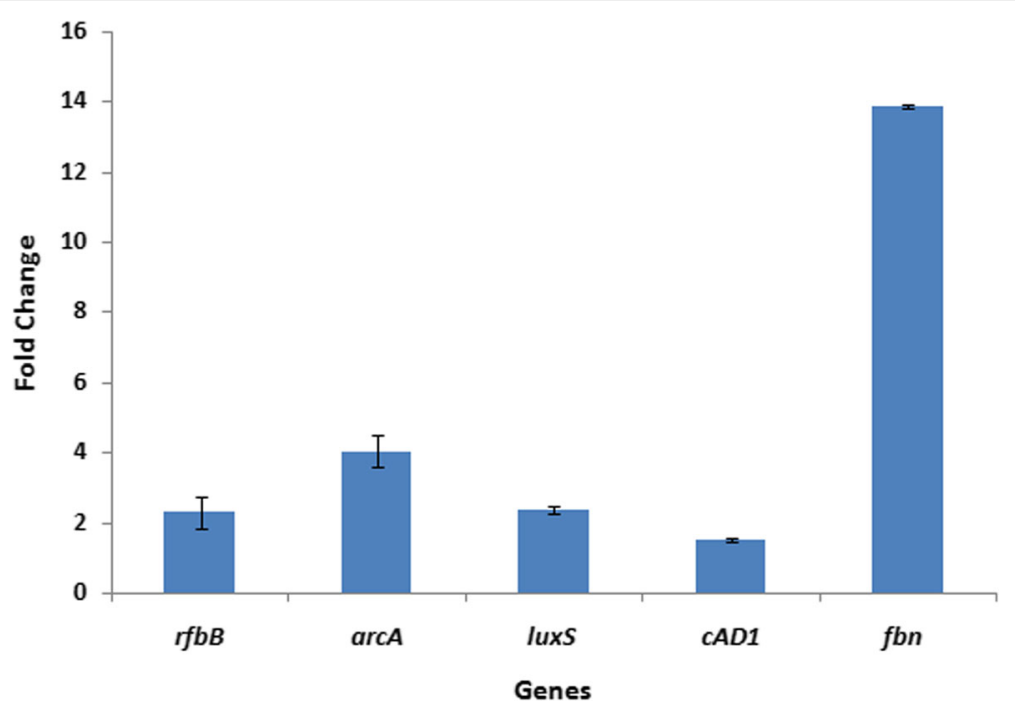

Fig. 4 Real time qPCR validation of selected biofilm associated genes. Fold change expression of genes observed in biofilm stage with respect to its corresponding planktonic stage

regulators and DNA binding response regulators have also shown an increasing trend in biofilm stages. The differential expression of major metabolic pathways and cellular processes upregulated in biofilm stages are discussed in this section.

\section{Pathways upregulated in biofilm stages Amino acid metabolism}

In the present investigation, various enzymes including chorismate synthase (3.97 fold), 3-phosphoshikimate 1carboxyvinyltransferase (1.5 fold), and 3-deoxy-7-phosphoheptulonate synthase (1.56 fold) which are involved in the biosynthesis of aromatic amino acids were found to be upregulated in biofilm stages. A similar trend of enhanced expression of aromatic amino acid biosynthesis enzymes has already been reported in a previous proteomic study performed on strong and weak biofilm forming E. faecalis strains [8]. Other major amino acid biosynthesis enzymes upregulated were Succinyl-diaminopimelate desuccinylase (6.35 fold), MTA/SAH nucleosidase (2.88 fold), cysteine synthase A (3.387 fold), Glutamine synthetase (5.81 fold), Acetolactate synthase (1.89 fold), etc. Previous studies on several other bacterial genera also revealed the role of amino acids in the formation of tight microcolonies and in maintaining the stability of mature biofilm [10-12]. Hence it is evident that during the biofilm maturation process, a series of amino acids and its related metabolites will be upregulated for contributing to extracellular matrix component of robust biofilms.

\section{Carbohydrate metabolism}

The present study evidenced the augmented production of seven major glycolytic enzymes ( 2.5 to 9 fold change) in biofilm stages compared to planktonic forms as listed in Table 1. Previous studies have shown the significant role of Glyceraldehyde 3-phosphate dehydrogenase in $P$. aeruginosa and Staphylococcus xylosus biofilms [13, 14] and also an upregulated expression in microaerophilic conditions in E.coli [15]. It is assumed that the cells within the biofilm will be in microaerophilic condition and needs a high level expression of these enzymes for adapting in the oxygen-limited environment. Another glycolytic enzyme phosphoglycerate mutase was found to be upregulated in S.xylosus biofilm [16] and is said to have a bifunctional role which helps in the synthesis of various EPSs, thus playing a significant role in the synthesis of core biofilm matrix [17]. Thus the glycolytic pathway is found to be one of the essential factors among biofilm survival mechanisms. Pyruvate dehydrogenase complex and several enzymes involved in Tricarboxylic acid (TCA) cycle and pentose phosphate pathways were also observed to be enhanced during the biofilm formation of SK460.

\section{Stress response factors and protein folding}

Several proteins involved in stress response including universal stress protein (10.69 fold), and General stress protein (3.42 fold) were identified to be steadily increased in biofilm stages. Similarly, universal stress proteins were found to be upregulated in Porphyromonas gingivalis and Sulfolobus solfataricus biofilms and showed impaired biofilm upon inactivation of these genes $[18,19]$. Signaling pathways for inducing stress response within the biofilm are cell-density dependent quorum sensing and the starvation-activated stringent response where the latter helps bacteria in adapting to 
nutrient deprivation. Nguyen et al. [20] identified the role of this stringent response in protecting bacteria within the biofilm from antimicrobial stress. It also activates the production of catalase and superoxide dismutase in stress condition. Enhanced expression of GTPase ObgE (1.53 fold) suggested the tendency of biofilm cells to attain the persistence nature in response to nutrient deprivation as suggested by previous findings [21]. Similarly, proteins like DPS family protein $(5.41$ fold), Thioredoxin family protein (4.75 fold), Alkyl hydroperoxide reductase (2.33 fold) and $\mathrm{H}_{2} \mathrm{O}_{2}$ scavenging proteins (9.11 fold) involved in reactive oxygen stress were also found to be over synthesized. It was also noticed that Gls24 is overexpressed by 9.2 fold in biofilm cells, suggesting its role in Enterococcal biofilm which is in accordance with the previous reports. Gls24 was found to be related to the stress and virulence of $E$. faecalis and was considered as a possible immunotherapy target [22].

Chaperonins and chaperones including GroL (1.63 fold), GroS (3.49 fold), several heat shock proteins, etc. were also found to be relatively abundant in SK460 biofilm. Enhanced levels of chaperones ensure the proper protein folding which enables E. faecalis to thrive within the biofilm. Clp proteases involved in the degradation of misfolded proteins also showed elevated expression in biofilm stages. Several studies have proved the role of chaperone DnaK in curlidependent biofilm formation and are considered as a potential target for anti-biofilm compounds [23-25].

\section{Rhamnose biosynthesis}

Rhamnose biosynthesis enzymes including glucose-1phosphate thymidylyltransferase (RfbA), dTDP-glucose 4,6-dehydratase (RfbB) and dTDP-4-dehydrorhamnose reductase (RfbD) were found to be more than threefold enhanced expression in the biofilm stage. Rhamnose biosynthesis enzymes belong to Enterococcal polysaccharide antigen (epa) gene cluster and were proved to have a major role in virulence in mouse peritonitis model $[26,27]$ and intestinal colonization [28]. This rhamnopolysaccharide epa also confers protection against high salt concentration and are said to be involved in osmotic stress response in E. faecalis [29]. Epa also confers resistance to various stresses including those of oxidative stress, stress towards ethanol, bile acids, detergent SDS and antimicrobial peptides. Decreased EPS production and colonization was observed due to knockout of rhamnose biosynthesis in Azospirillum brasilense [30]. Thus rhamnopolysaccharide serves as a major extracellular matrix component as well as enables the cells to survive within the stressed environment of the matrix.

\section{Arginine metabolism}

Arginine deiminase (ArcA), ornithine carbamoyltransferase $(\mathrm{ArcB})$ and carbamate kinase (ArcC) are enzymes involved in arginine deiminase (ADI) pathway and were found to be highly expressed (ranging from 1.9 to 8 fold) in E. faecalis biofilm in the present study. Ammonia synthesis via the ADI pathway is important to reduce the $\mathrm{pH}$ stress within the microcolonies. Mutants of arginine deiminase have showed decreased viability in S. epidermidis biofilm, and ADI is proved to have a role in $\mathrm{pH}$ homeostasis in biofilms [31]. Transcription of arc operon is often arginine dependent via ArgR family of regulators (ArgR and $\mathrm{AhrC}$ ) which were found to be active in biofilm stages. ADI pathway is generally induced under anaerobic conditions and hence this may have a role in the oxygen reduced environment within the biofilm.

\section{Quorum sensing (QS) and pheromone associated proteins} S-ribosylhomocysteinase (LuxS) was shown to have a rising trend with more than threefold increased expression in biofilm stages. Previous studies evidenced that luxS mutants developed an altered biofilm and exhibited enhanced cell-surface hydrophobicity in E. faecalis [32]. Several other studies also had a similar observation of the negative impact of luxS mutants in Streptococcus anginosus, and S. mutans biofilms [33, 34]. But still, the role of luxS dependent quorum sensing is not well established in E. faecalis biofilm.

Pheromone cAD1 lipoprotein (1.91 fold) and other pheromone associated proteins were upregulated in biofilm stages. Surface-associated pheromone binding lipoproteins act as essential components for adhesion, colonization and virulence and are also involved in stress response. Varahan et al. [35] showed that pheromone transporter mutants displayed altered biofilm architecture with a significant reduction in biofilm biomass compared to wild type suggesting the role of pheromones and associated proteins in biofilm development.

\section{Adhesion associated proteins}

Aggregation substance was already proved to have a role in Enterococcal biofilm formation [3, 36]. Adhesion lipoproteins are surface-associated lipoproteins involved in Enterococcal adherence, colonization and virulence [37] and are directly linked to biofilm formation as in agreement with our findings. Fibronectin/fibrinogen binding proteins also showed more than tenfold increase in biofilm stages and were suggested to have a significant role in E. faecalis associated urinary tract infections in a murine model [38] and endocarditis in rats [39]. Fibronectin binding protein mutants of community-associated MRSA losses the ability for fibronectin binding thereby preventing the establishment of biofilm in host tissue [40]. These proteins were assumed to be associated with bacterial aggregation and involve in primary attachment 
to a surface and hence can be considered as a suitable biofilm inhibiting target.

\section{Other major factors}

Several DNA binding response regulators, phosphotransferase system transporter proteins, Autolysin, Dalanylation of lipoteichoic acid (dltABCD), etc. were also found to be more than two-fold upregulated in biofilm stages. Of these, autolysin is well known to be associated with the eDNA release in the matrix and structural integrity of the biofilm. Previous report showed that autolysin deficient mutants had defects in primary attachment and eDNA release which are mainly associated with the accumulative phase for maturation and structural stability of biofilm in E. faecalis [5]. These mutants lack DNase1-sensitive fibrous network which is found to have a role in biofilm stability. D-alanylation of lipoteichoic acid ( $\operatorname{dlt} C$ and $d l t D$ ) was also found to be abundant in biofilm mode (2.36 to 4.17 fold), possibly incorporating lipoteichoic acid into the EPS matrix. Lack of d-alanine esters results in a stronger negative net charge on the bacterial cell surface and dlt mutants showed a reduction in biofilm formation on polystyrene surfaces. Furthermore, dltABCD operon is involved in the pathogenesis of $E$. faecalis leading to enhanced biofilm formation, host tissue attachment and increased resistance to antimicrobial peptides [6].

STRING analysis revealed that upregulated genes including stress response factors, major glycolytic enzymes, arginine metabolism and rhamnose biosynthesis were either directly or indirectly poses a close molecular interaction ( $p$-value $<1.0 \mathrm{e}-16)$, thereby regulating each other in stressful environment prevailing during biofilm development. Among the upregulated cellular processes, $r f b B$ (Rhamnose biosynthesis), $\operatorname{arcA}$ (Arginine deiminase), luxS (Quorum sensing), $c A D 1$ (Pheromone cAD1 lipoprotein) and $f b n$ (Fibrinogen/Fibronectin binding protein associated with adhesion) were selected for gene expression analysis and were found to be enhanced by $2.29,4.03,2.35,1.5$ and 13.87 fold respectively in biofilm stages. Of these, luxS mediated quorum sensing system is attributed to play a major role in E. faecalis SK460 biofilm which is devoid of fsr two-component signal transduction system. Accordance of the proteome data with RT-PCR results confirms the reliability of the analysis, serving as a validation for the identified determinants of Enterococcal biofilm development.

\section{Conclusion}

In E. faecalis, comparative proteome analysis revealed that pathways belonging to carbohydrate metabolism including glycolysis and pyruvate metabolism, amino acid metabolism, biosynthesis of secondary metabolites and microbial metabolism in diverse environments comprising stress response factors were upregulated in biofilm stages. Stress response factors are major groups enabling the biofilm cells to resist the $\mathrm{pH}$ stress, oxidative stress and nutrient deprivation. Proteome analysis also elucidated interesting observations on the relevance of luxS mediated quorum sensing and pheromone associated proteins in biofilm development of fsr negative non-gelatinase producing Enterococci. The high-level expression of rhamnose biosynthesis genes proposes the role of rhamnopolysaccharide as a major component strengthening the biofilm EPS matrix. In the present study, all the major pathways found to be associated with the biofilm phenotype might be playing an inevitable role in biofilm associated infections. Indeed, the generated proteome data had provided a set of interesting targets in the biofilm forming mechanism of E. faecalis. Future gene mutation studies are warranted to prove the exact role of these factors in biofilm establishment. The identified targets along with the advanced computational drug discovery approaches and high-throughput screening would help to accelerate the development of suitable anti-biofilm agents against Enterococcal biofilm.

\section{Methods}

\section{Bacterial strain and growth condition}

E. faecalis SK460, isolated from chronic diabetic ulcer patient was used for quantitative proteomics approach to identify biofilm-associated proteins. The study was approved by the Institutional Human Ethics Committee (Reference number RGCB-IEC No. IHEC/01/2013/11) of Rajiv Gandhi Centre for Biotechnology, Kerala, India. The draft genome sequence of this strain was deposited at NCBI Genbank with accession no. NIXL00000000 [41]. The strain was cultured in Luria Bertani broth (Becton Dickinson, New Jersey, United States) at $37^{\circ} \mathrm{C}$ with $150 \mathrm{rpm}$. The media used for culturing biofilm was Tryptic soy broth (Himedia, Mumbai, India) with $0.75 \%$ Glucose.

\section{Biofilm quantification and imaging}

For confocal imaging, an overnight culture of E. faecalis SK460 was diluted to OD600 $=0.1$ and $4 \mathrm{ml}$ of diluted broth was distributed to 6 well microtitre plate (Nunc, Roskilde, Denmark). A sterile square glass coverslip was inserted horizontally in each well and incubated at $37^{\circ} \mathrm{C}$ in a static condition for two-time intervals $(12 \mathrm{~h}, 24 \mathrm{~h})$. Biofilm staining was carried out with Syto9 (Invitrogen, California, United States) as described previously [42]. The slides were then observed using a Nikon Eclipse Ti Confocal Laser scanning inverted microscope (Nikon Instruments Corporation, New York, United States). The measurement of biofilm thickness was performed using NIS-Element AR software, version 4.00.04. 


\section{Cell extract preparation}

The strain was cultured in different planktonic and biofilm stages to carry out quantitative proteome analysis. The test strain was inoculated in Tryptic soy broth (with $0.75 \%$ glucose) and incubated at $37{ }^{\circ} \mathrm{C}$ with shaking at $180 \mathrm{rpm}$. After overnight incubation, the culture was diluted to adjust the optical density to 0.1 at $600 \mathrm{~nm}$.

\section{Harvesting planktonic stage cells}

One hundred milliliter of diluted culture was dispensed each in $250 \mathrm{ml}$ conical flasks for two different time points $(12 \mathrm{~h}, 24 \mathrm{~h})$ in triplicates and incubated at $37^{\circ} \mathrm{C}$ with shaking at $150 \mathrm{rpm}$. After proper incubation, the cells were pelleted and washed twice with sterile ice-cold phosphate buffered saline (PBS, $\mathrm{pH} 7.4$ ) and stored at $-80^{\circ} \mathrm{C}$ till protein isolation.

\section{Harvesting biofilm stage cells}

Similarly, the diluted culture was dispensed to Cell Culture Treated EasYFlasks, $175 \mathrm{~cm}^{2}$ (Nunc, Roskilde, Denmark) at two-time points $(12 \mathrm{~h}, 24 \mathrm{~h})$ in triplicates and incubated at $37^{\circ} \mathrm{C}$ in a static condition. After incubation, the used media along with the planktonic cells were washed off and the flask was rinsed twice with sterile PBS to remove the settled planktonic cells. The biofilm cells were then scraped off, pooled in sterile icecold PBS, pelleted and stored at $-80^{\circ} \mathrm{C}$ till protein isolation.

\section{Protein sample preparation}

Protein isolation from cell pellets of different planktonic and biofilm stages was carried out by bead beating as described previously [43]. Briefly, cell pellets were resuspended in PBS (1 ml PBS per gram of cells) and transferred to $2 \mathrm{ml}$ microcentrifuge tubes containing glass beads $(0.5 \mathrm{~mm})$ and protease inhibitor $(1 \mathrm{mM}$ Phenylmethyl Sulfonyl Fluoride, PMSF) and incubated on ice for $5 \mathrm{~min}$. The tubes were then placed in a Mini Bead beater (BioSpec Products, USA) and subjected to one-minute pulses at $4200 \mathrm{rpm}$ for three times with 1 min interval. The suspensions were centrifuged at 13 , $000 \mathrm{rpm}$ for $10 \mathrm{~min}$ at $4{ }^{\circ} \mathrm{C}$ and the supernatant was collected. Protein concentration was estimated by bicinchoninic acid protein assay kit as followed by the manufacturer's instructions (Thermo Scientific, Massachusetts, United States). Three biological replicates and three technical replicates were used in this experiment. $100 \mu \mathrm{g}$ of protein $(1 \mu \mathrm{g} / \mu \mathrm{l})$ from each sample was made up to $100 \mu \mathrm{l}$ with $50 \mathrm{mM}$ Ammonium bicarbonate and subjected to disulfide reduction, alkylation and insolution tryptic digestion as followed by previous publication [44]. The digested peptide solution was centrifuged at maximum $\mathrm{rpm}$ at $4{ }^{\circ} \mathrm{C}$ for $12 \mathrm{~min}$ and the supernatant was stored at $-20^{\circ} \mathrm{C}$ until the analysis using
Liquid Chromatography-Tandem Mass Spectrometry (LC-MS/MS).

\section{LC-MS/MS analysis}

Peptide samples were analyzed by nano-LC/MS $\mathrm{E}_{\mathrm{E}}$ (at elevated energy) using a nanoACQUITY UPLC ${ }^{\ominus}$ system (Waters, Altrincham, UK) coupled to a QuadrupoleTime of Flight $(\mathrm{Q} / \mathrm{TOF})$ mass spectrometer (SYNAPTG2, Waters, Altrincham, UK). Instrument operation and control for both the system was done employing MassLynx4.1 SCN781 software. The peptides were separated by reverse-phase column chromatography in the nanoACQUITY UPLC ${ }^{\circ}$. Peptides eluted from the nanoLC were subjected to mass spectrometric analysis on a SYNAPT $^{\circ}$ G2 High Definition MS ${ }^{\mathrm{m}}$ System (Waters, Altrincham, UK). All analyses were performed using positive mode ESI using a NanoLockSpray ${ }^{\text {mim }}$ source. The detailed operational set up was followed as in previous publications [44].

\section{Data analysis}

ProteinLynx Global SERVER ${ }^{\mathrm{m}}$ (PLGS) v2.5.3 (Waters, Altrincham, UK) was used to analyze the acquired ion mobility enhanced $\mathrm{MS}^{\mathrm{E}}$ spectra for protein identification as well as for the label-free relative protein quantification. Data processing and parameter setups were carried out as followed by [44]. The sequence database of the reference strain E. faecalis V583 (NCBI Reference Sequence: NC_004668.1) was used for database search. The false positive rate (FPR) was set to $4 \%$ with a randomized database, appended to the original one. The parameters for protein identification were made in such a way that a peptide was required to have at least one fragment ion match, a protein was required to have at least three fragment ion matches, and two peptide matches for identification. The peptides with $50 \%$ or more probability to be present in the mixture and detected with a score above 20 , as calculated by the software were selected for proteomic analysis [45]. Data sets were normalized using the 'internal standard-normalization' function of PLGS and label-free quantitative analysis was performed by comparing the normalized peak area/intensity of identified peptides between the samples.

\section{Analysis of physicochemical properties of identified proteome}

The hydrophobicity of the identified proteins was calculated based on grand average hydropathy (GRAVY) value using the online GRAVY calculator (http://www.gravycalculator.de/). The GRAVY score was calculated by dividing the sum of hydropathy values of all the amino acids by the protein length [46]. Isoelectric point (pI) and Molecular weight (MW) of the proteins was calculated 
using compute $\mathrm{pI} / \mathrm{MW}$ tool of ExPASy Bioinformatics Resource Portal (https://web.expasy.org/compute_pi/).

\section{Gene ontology analysis}

The total proteins from all the stages were analyzed using the Venny tool 2.1.0 to identify proteins specific to the planktonic and biofilm stages. The total proteome of the planktonic stage was obtained by compiling the total proteins identified in the triplicates of two different time points of planktonic stage viz., $12 \mathrm{~h}$ and $24 \mathrm{~h}$. Similarly, the total proteome of biofilm stages was compiled. The identified proteins were subjected to Gene Ontology (GO) and Kyoto Encyclopedia of Gene and Genomes (KEGG) analysis using Database for Annotation, Visualization and Integrated Discovery (DAVID) functional Annotation Bioinformatic analyzer [47, 48]. DAVID generates Expression Analysis Systematic Explorer (EASE) score, a modified Fisher's Exact $P$-value for each term and the GO and KEGG pathway terms with a $P$ value $\leq 0.1$ was considered as enriched. Average relative expression values of at least two biological replicates were used and a fold change of more than 30\% (ratio of either $<0.70$ for downregulation and $>1.3$ for upregulation) was considered to be significantly altered levels of expression. Protein-protein interaction networks of differentially overexpressed proteins in biofilm stages were generated using STRING 10.5 (https://string-db.org/) [49] and a confidence view was generated by setting the filter to medium confidence (0.400).

\section{Real-time PCR analysis}

RNA was isolated from planktonic and biofilm stages of E. faecalis SK460 using RNeasy mini kit (Qiagen, Hilden, Germany) as per manufacturer's protocol involving lysis with lysozyme and finally treated with DNase-1 (SigmaAldrich, Missouri, United States). Conventional PCR using 16SrRNA gene was performed to check the DNA contamination. cDNA was synthesized using Prime Script $^{\text {ti }} 1$ st strand cDNA synthesis kit according to the manufacturer's protocol (Applied Biosystems, California, United States) and stored at ${ }^{-} 20^{\circ} \mathrm{C}$.

Primers of selected genes were designed using PrimerQuest tool of Integrated DNA Technologies (Integrated DNA Technologies, Inc., California, US) and are shown in Additional file 1: Table S1. Power SYBR Green PCR Master Mix (Takara, Kusatsu, Japan) was used for realtime PCR in Applied Biosystems 7900HT Fast RealTime PCR System as per the manufacturer's suggestion. The reaction procedure was as follows: incubation at $95^{\circ} \mathrm{C}$ for $10 \mathrm{~min}$ followed by 40 cycles of $95^{\circ} \mathrm{C}$ for $10 \mathrm{~s}$, $49^{\circ} \mathrm{C}$ for $15 \mathrm{~s}$ and $60^{\circ} \mathrm{C}$ for $45 \mathrm{~s}$. Three independent reactions were conducted with triplicates for each gene and reaction mixture without RNA template was used as negative control for each set of primers. Expression analysis of each gene was calculated based on at least two independent experiments. Melting curve analysis was done to verify primer specificity and relative expression values were calculated as $2-\Delta$ (CT Target - CT reference) with 23S rRNA gene as housekeeping gene.

\section{Additional files}

\begin{abstract}
Additional file 1: Table S1. List of primers used for qRT-PCR (DOC $35 \mathrm{~kb}$ )
Additional file 2: Figure S1. a) Scatter plot showing the distribution of GRAVY index for the proteins identified in planktonic and biofilm stages. b). Distribution of the predicted molecular weight (MW) and isoelectric point (pl) for the total proteins obtained in planktonic and biofilm stages. (TIF 8548 kb)

Additional file 3: Figure S2. Functional interactome of up-regulated proteins in biofilm stages, generated using STRING network prediction algorithm (Version 10.5). Colored nodes: query proteins and first shell of interactors. Empty nodes: proteins of unknown 3D structure. Filled nodes: some 3D structure is known or predicted. Edges represent proteinprotein associations. Known interactions- Pink represents experimentally determined, wathet-blue represents from curated database, predicted interactions -green represents gene neighborhood, dark blue represents gene co-occurrence, red represents gene fusions, and black represents co-expression. (TIF $8948 \mathrm{~kb}$ )
\end{abstract}

\section{Abbreviations}

DAVID: Database for Annotation, Visualization and Integrated Discovery; EASE: Expression Analysis Systematic Explorer; EPS: Extra polymeric substance; FPR: False positive rate; fsr: Fecal Streptococci regulator; GO: Gene Ontology; KEGG: Kyoto Encyclopedia of Gene and Genomes; LC-MS/MS: Liquid Chromatography - Tandem Mass Spectrometry; LuxS: Sribosylhomocysteinase; PBS: Phosphate buffered saline; PLGS: ProteinLynx Global SERVER ${ }^{\text {TM }}$

\section{Acknowledgments}

The authors would like to thank Prof. M. Radhakrishna Pillai, Director, Rajiv Gandhi Centre for Biotechnology, for providing the necessary facilities. We also acknowledge Arun Surendran and Mahesh Chandran at the Mass Spectrometry and Proteomics Core Facility for technical assistance in LC-MS/ MS analysis and also members of Confocal Imaging Core Facility for assistance in imaging. KS acknowledges Kerala State Council for Science, Technology \& Environment, Government of Kerala for the fellowship provided.

\section{Authors' contributions}

KS and ST conceived and designed the experiments. KS and LN performed the experiments, KS, JJ, SG and ST analyzed the results. KS and MPR drafted the manuscript. ST critically reviewed the manuscript. All authors read and approved the final manuscript.

\section{Funding}

This work is funded by Department of Biotechnology, Ministry of Science and Technology, Govt. of India (Grant no. BT/PR15261/MED/29/994/2015). The funding body had no role in the design of the study, analysis and interpretation of data and in writing the manuscript.

\section{Availability of data and materials}

The mass spectrometry proteomics data have been deposited to the ProteomeXchange Consortium via the PRIDE partner repository with the dataset identifier PXD010770.

\section{Ethics approval and consent to participate}

The study was approved by the Institutional Human Ethics Committee (Reference number RGCB-IEC No. IHEC/01/2013/11) of Rajiv Gandhi Centre for Biotechnology. The participants were enrolled in this study after signing the informed consent protocol form. Animal Ethics clearance is not applicable, as this study does not involve any animals. 


\section{Consent for publication}

Not applicable.

\section{Competing interests}

The authors declare that they have no competing interests.

\section{Author details}

${ }^{1}$ Cholera and Biofilm Research Laboratory, Pathogen Biology, Rajiv Gandhi Centre for Biotechnology, (National Institute under the Department of Biotechnology, Government of India), Trivandrum, Kerala 695014, India. ${ }^{2}$ Department of Surgery, Government Medical College Hospital, Trivandrum, Kerala 695011, India. Interdisciplinary Biology, Rajiv Gandhi Centre for Biotechnology, Trivandrum, Kerala 695014, India.

\section{Received: 25 April 2019 Accepted: 20 June 2019}

Published online: 28 June 2019

\section{References}

1. Hancock LE, Perego M. Systematic inactivation and phenotypic characterization of two-component signal transduction systems of Enterococcus faecalis V583. J Bacteriol. 2004;186(23):7951-8. https://doi.org/ 10.1128/JB.186.23.7951-7958.

2. Tendolkar PM, Baghdayan AS, Gilmore MS, Shankar N. Enterococcal surface protein, Esp, enhances biofilm formation by Enterococcus faecalis. Infect Immun. 2004:72(10):6032-9. https://doi.org/10.1128/IAl.72.10.6032-6039.2004.

3. Chuang-Smith ON, Wells CL, Henry-Stanley MJ, Dunny GM. Acceleration of Enterococcus faecalis biofilm formation by aggregation substance expression in an ex vivo model of cardiac valve colonization. PLoS One. 2010;5(12): e15798. https://doi.org/10.1371/journal.pone.0015798.

4. Bourgogne A, Singh KV, Fox KA, Pflughoeft KJ, Murray BE, Garsin DA. EbpR is important for biofilm formation by activating expression of the endocarditis and biofilm-associated pilus operon (ebpABC) of Enterococcus faecalis OG1RF. J Bacteriol. 2007;189(17):6490-3. https://doi.org/10.1128/JB.00594-07.

5. Guiton PS, Hung CS, Kline KA, Roth R, Kau AL, Hayes E, Heuser J, Dodson KW, Caparon MG, Hultgren SJ. Contribution of autolysin and sortase a during Enterococcus faecalis DNA-dependent biofilm development. Infect Immun. 2009;77(9):3626-38. https://doi.org/10.1128/IAl.00219-09.

6. Fabretti F, Theilacker C, Baldassarri L, Kaczynski Z, Kropec A, Holst O, Huebner J. Alanine esters of enterococcallipoteichoic acid play a role in biofilm formation and resistance to antimicrobial peptides. Infect Immun. 2006;74(7):4164-71. https://doi.org/10.1128/IAl.00111-06.

7. Qayyum S, Sharma D, Bisht D, Khan AU. Protein translation machinery holds a key for transition of planktonic cells to biofilm state in Enterococcus faecalis: a proteomic approach. Biochem Biophys Res Commun. 2016;474(4): 652-9. https://doi.org/10.1016/j.bbrc.2016.04.145.

8. $\quad$ Suriyanarayanan T, Qingsong L, Kwang LT, Mun LY, Seneviratne CJ. Quantitative proteomics of strong and weak biofilm formers of Enterococcus faecalis reveals novel regulators of biofilm formation. Mol Cell Proteomics. 2018;17(4):643-54. https://doi.org/10.1074/mcp.RA117.000461.

9. Suryaletha K, Narendrakumar L, John J, Reghunathan D, Prasannakumar M, Thomas S. Genomic insights into a biofilm forming multidrug resistant Enterococcus faecalis SK460 isolated from a chronic diabetic ulcer patient. Genome Announc. 2018;6(2):e01463-17. https://doi.org/10.1128/genomeA. 01463-17.

10. Sriramulu DD, Lünsdorf H, Lam JS, Römling U. Microcolony formation: a novel biofilm model of Pseudomonas aeruginosa for the cystic fibrosis lung. J Med Microbiol. 2005;54(pt7):667-76. https://doi.org/10.1099/jmm. 0.45969-0.

11. Losensky G, Jung K, Urlaub H, Pfeifer F, Fröls S, Lenz C. Shedding light on biofilm formation of Halobacterium salinarum R1 by SWATH-LC/MS/MS analysis of planktonic and sessile cells. Proteomics. 2017;17(7). https://doi. org/10.1002/pmic.201600111.

12. Mohammed MMA, Pettersen VK, Nerland AH, Wiker HG, Bakken V. Quantitative proteomic analysis of extracellular matrix extracted from mono- and dual-species biofilms of Fusobacterium nucleatum and Porphyromonas gingivalis. Anaerobe. 2017:44:133-42. https://doi.org/10. 1016/j.anaerobe.2017.03.002.

13. Sauer K, Camper AK, Ehrlich GD, Costerton JW, Davies DG. Pseudomonas aeruginosa displays multiple phenotypes during development as a biofilm. J Bacteriol. 2002;184:1140-54. https://doi.org/10.1128/jb.184.4.1140-1154.2002.
14. Planchon S, Desvaux M, Chafsey I, Chambon C, Leroy S, Hébraud M, Talon R. Comparative subproteome analyses of planktonic and sessile Staphylococcus xylosus C2a: new insight in cell physiology of a coagulase-negative Staphylococcus in biofilm. J Proteome Res. 2009;8(4):1797-809. https://doi. org/10.1021/pr8004056.

15. Peng L, Shimizu K. Global metabolic regulation analysis for Escherichia coli K12 based on protein expression by 2-dimensional electrophoresis and enzyme activity measurement. Appl Microbiol Biotechnol. 2003;61(2):163-78. https://doi.org/10.1007/s00253-002-1202-6.

16. Planchon S, Chambon C, Desvaux M, Chafsey I, Leroyt S, Talon R, Hébraud M. Proteomic analysis of cell envelope from Staphylococcus xylosus C2a, a coagulase-negative Staphylococcus. J Proteome Res. 2007;6(9):3566-80. https://doi.org/10.1021/pr070139.

17. Levander F, Rådström P. Requirement for phosphoglucomutase in exopolysaccharide biosynthesis in glucose- and lactose-utilizing Streptococcus thermophilus. Appl Environ Microbiol. 2001;67(6):2734-8. https://doi.org/10.1128/AEM.67.6.2734-2738.2001.

18. Chen W, Honma K, Sharma A, Kuramitsu HK. A universal stress protein of Porphyromonas gingivalis is involved in stress responses and biofilm formation. FEMS Microbiol Lett. 2006;264(1):15-21. https://doi.org/10.1111/j. 1574-6968.2006.00426.x.

19. Koerdt A, Orell A, Pham TK, Mukherjee J, Wlodkowski A, Karunakaran E, Biggs CA, Wright PC, Albers SV. Macromolecular fingerprinting of Sulfolobus species in biofilm: a transcriptomic and proteomic approach combined with spectroscopic analysis. J Proteome Res. 2011;10(9):4105-19. https://doi.org/ 10.1021/pr2003006

20. Nguyen D, Joshi-Datar A, Lepine F, Bauerle E, Olakanmi O, Beer K, McKay G, Siehnel R, Schafhauser J, Wang Y, Britigan BE, Singh PK. Active starvation responses mediate antibiotic tolerance in biofilms and nutrient-limited bacteria. Science. 2011;334(6058):982-6. https://doi.org/10.1126/science. 1211037.

21. Verstraeten N, Knapen WJ, Kint Cl, Liebens V, Van den Bergh B, Dewachter L, Michiels JE, Fu Q, David CC, Fierro AC, Marchal K, Beirlant J, Versées W, Hofkens J, Jansen M, Fauvart M, Michiels J. Obg and membrane depolarization are part of a microbial bet-hedging strategy that leads to antibiotic tolerance. Mol Cell. 2015;59(1):472-80. https://doi.org/10.1016/j. molcel.2015.05.011.

22. Teng F, Nannini EC, Murray BE. Importance of gls24 in virulence and stress response of Enterococcus faecalis and use of the Gls24 protein as a possible immunotherapy target. J Infect Dis. 2005;191(3):472-80. https://doi.org/10. 1086/427191.

23. Andersson EM, Andersson EK, Bengtsson C, Evans ML, Chorell E, Sellstedt M, Lindgren AEG, Hufnagel DA, Bhattacharya M, Tessier PM, Wittung-Stafshede P, Almqvist F, Chapman MR. Modulation of Curli assembly and pellicle biofilm formation by chemical and protein chaperones. Chem Biol. 2013; 20(10):1245-54. https://doi.org/10.1016/j.chembiol.2013.07.017.

24. Arita-Morioka K, Kunitoshi Y, Mizunoe Y, Ogura T, Sugimoto S. Novel strategy for biofilm inhibition by using small molecules targeting molecular chaperone DnaK. Antimicrob Agents Chemother. 2015;59(1):633-41. https:// doi.org/10.1128/AAC.04465-14.

25. Sugimoto S, Arita-Morioka K, Terao A, Yamanaka K, Ogura T, Mizunoe Y. Multitasking of $\mathrm{Hsp70}$ chaperone in the biogenesis of bacterial functional amyloids. Commun Biol. 2018;1:52. https://doi.org/10.1038/ s42003-018-0056-0.

26. Xu Y, Barbara EM, George MW. A cluster of genes involved in polysaccharide biosynthesis from Enterococcus faecalis OG1RF. Infect Immun. 1998;66:4313-23.

27. Teng F, Singh KV, Bourgogne A, Zeng J, Murray BE. Further characterization of the epa gene cluster and Epa polysaccharides of Enterococcus faecalis. Infect Immun. 2009;77(9):3759-67. https://doi.org/10.1128/IAl.00149-09.

28. Rigottier-Gios L, Madec C, Navickas A, Matos RC, Akary-Lepage E, Mistou MY, Serror P. The surface Rhamnopolysaccharide Epa of Enterococcus faecalis is a key determinant of intestinal colonization. J Infect Dis. 2015;211(1):62-71. https://doi.org/10.1093/infdis/jiu402.

29. Solheim M, La Rosa SL, Mathisen T, Snipen LG, Nes IF, Brede DA. Transcriptomic and functional analysis of $\mathrm{NaCl}$-induced stress in Enterococcus faecalis. PLoS One. 2014;9(4):e94571. https:/doi.org/10.1371/journal.pone.0094571.

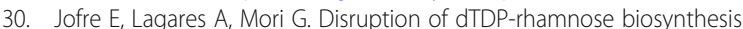
modifies lipopolysaccharide core, exopolysaccharide production and root colonization in Azospirillum brasilense. FEMS Microbiol Lett. 2004;231(2): 267-75. https://doi.org/10.1016/S0378-1097(04)00003-5. 
31. Lindgren JK, Thomas VC, Olson ME, Chaudhari SS, Nuxoll AS, Schaeffer CR, Lindgren KE, Jones J, Zimmerman MC, Dunman PM, et al. Arginine deiminase in Staphylococcus epidermidis functions to augment biofilm maturation through pH homeostasis. J Bacteriol. 2014;196(12):2277-89. https://doi.org/10.1128/JB.00051-14.

32. He Z, Liang J, Zhou W, Xie Q, Tang Z, Ma R, Huang Z. Effect of the quorumsensing luxS gene on biofilm formation by Enterococcus faecalis. Eur J Oral Sci. 2016;124(3):234-40. https://doi.org/10.1111/eos.12269.

33. Petersen FC, Ahmed NA, Naemi A, Scheie AA. LuxS-mediated signaling in Streptococcus anginosus and its role in biofilm formation. Antonie Van Leeuwenhoek. 2006;90:109-21. https://doi.org/10.1007/s10482-006-9065-y.

34. Yoshida A, Ansai T, Takehara T, Kuramitsu HK. LuxS-based signaling affects Streptococcus mutans biofilm formation. Appl Environ Microbiol. 2005;71: 2372-80. https://doi.org/10.1128/AEM.71.5.2372-2380.2005.

35. Varahan S, Harms N, Gilmore MS, Tomich JM, Hancock LE. An ABC transporter is required for secretion of peptide sex pheromones in Enterococcus faecalis. Mbio. 2014;5(5):e01726-14. https://doi.org/10.1128/ mBio.01726-14.

36. Bhatty M, Cruz MR, Frank KL, Gomez JA, Andrade F, Garsin DA, Dunny GM, Kaplan HB, Christie PJ. Enterococcus faecalis pCF10-encoded surface proteins PrgA, PrgB (aggregation substance) and PrgC contribute to plasmid transfer, biofilm formation and virulence. Mol Microbiol. 2015;95(4):660-77. https:// doi.org/10.1111/mmi.12893.

37. Reffuveille F, Leneveu C, Chevalier S, Auffray Y, Rince A. Lipoproteins of Enterococcus faecalis: bioinformatic identification, expression analysis and relation to virulence. Microbiology. 2011;157(pt11):3001-13. https://doi.org/ 10.1099/mic.0.053314-0.

38. Torelli R, Serror P, Bugli F, Paroni SF, Florio AR, Stringaro A, Colone M, De Carolis E, Martini C, Giard JC, et al. The PavA-like fibronectin-binding protein of Enterococcus faecalis, EfbA, is important for virulence in a mouse model of ascending urinary tract infection. J Infect Dis. 2012;206(6):952-60. https:// doi.org/10.1093/infdis/jis440.

39. Singh KV, Larose SL, Somarajan SR, Roh JH, Murray B. The fibronectinbinding protein EfbA contributes to pathogenesis and protects against infective endocarditis caused by Enterococcus faecalis. Infect Immun. 2015; 83(12):4487-94. https://doi.org/10.1128/IAl.00884-15.

40. McCourt J, O'Halloran DP, McCarthy H, O'Gara JP, Geoghegan JA. Fibronectin-binding proteins are required for biofilm formation by community-associated methicillin-resistant Staphylococcus aureus strain LAC. FEMS Microbiol Lett. 2014;353(2):157-64. https://doi.org/10.1111/1574-6968. 12424.

41. Suryaletha K, John J, Radhakrishnan MP, George S, Thomas S. Metataxonomic approach to decipher the polymicrobial burden in diabetic foot ulcer and its biofilm mode of infection. Int Wound J. 2018;15(3):473-81. https://doi.org/10.1111/iwj.12888.

42. Cerca N, Martins S, Sillankorva S, Jefferson KK, Pier GB, Oliveira R, Azeredo J. Effects of growth in the presence of subinhibitory concentrations of dicloxacillin on Staphylococcus epidermidis and Staphylococcus haemolyticus biofilms. Appl Environ Microbiol. 2005;71(12):8677-82. https://doi.org/10. 1128/AEM.71.12.8677-8682.2005.

43. Gopinath V, Raghunandanan S, Gomez RL, Jose L, Surendran A, Ramachandran R, Pushparajan AR, Mundayoor S, Jaleel A, Kumar RA. Profiling the proteome of Mycobacterium tuberculosis during dormancy and reactivation. Mol Cell Proteomics. 2015;14(8):2160-76. https://doi.org/10. 1074/mcp.M115.051151.

44. Dharmaprakash A, Mutt E, Jaleel A, Ramanathan S, Thomas S. Proteome profile of a pandemic Vibrio parahaemolyticus SC192 strain in the planktonic and biofilm condition. Biofouling. 2014;30(6):729-39. https://doi.org/10.1080/ 08927014.2014.916696

45. Cockman ME, Webb JD, Kramer HB, Kessler BM, Ratcliffe PJ. Proteomicsbased identification of novel factor inhibiting hypoxia-inducible factor $(F \mid H)$ substrates indicates widespread asparaginyl hydroxylation of ankyrin repeat domain-containing proteins. Mol Cell Proteomics. 2009;8(3):535-46. https:// doi.org/10.1074/mcp.M800340-MCP200.

46. Molloy MP, Herbert BR, Slade MB, Rabilloud T, Nouwens AS, Williams KL, Gooley AA. Proteomic analysis of the Escherichia coli outer membrane. Eur J Biochem. 2000;267(10):2871-81. https://doi.org/10.1046/j.1432-1327.2000. 01296.x.

47. Huang DW, Sherman BT, Lempicki RA. Systematic and integrative analysis of large gene lists using DAVID bioinformatics resources. Nat Protoc. 2009;4(1): 44-57. https://doi.org/10.1038/nprot.2008.211.
48. Huang DW, Sherman BT, Lempicki RA. Bioinformatics enrichment tools: paths toward the comprehensive functional analysis of large gene lists. Nucleic Acids Res. 2009;37(1):1-13. https://doi.org/10.1093/nar/gkn923.

49. Szklarczyk D, Franceschini A, Wyder S, Forslund K, Heller D, Huerta-Cepas J, Simonovic M, Roth A, Santos A, Tsafou KP, et al. STRING v10: protein-protein interaction networks, integrated over the tree of life. Nucleic Acids Res. 2015;43:D447-52. https://doi.org/10.1093/nar/gku1003.

\section{Publisher's Note}

Springer Nature remains neutral with regard to jurisdictional claims in published maps and institutional affiliations.
Ready to submit your research? Choose BMC and benefit from:

- fast, convenient online submission

- thorough peer review by experienced researchers in your field

- rapid publication on acceptance

- support for research data, including large and complex data types

- gold Open Access which fosters wider collaboration and increased citations

- maximum visibility for your research: over $100 \mathrm{M}$ website views per year

At BMC, research is always in progress.

Learn more biomedcentral.com/submissions 